\title{
NOTE ON THE NONEXISTENCE OF MINIMAL SURFACES
}

\author{
JOHANNES C. C. NITSCHE ${ }^{1}$
}

While a Jordan curve in Euclidean 3-space always bounds a minimal surface of disc-type, it is generally quite difficult to decide whether a frame of several contours is capable of bounding a minimal surface having a prescribed topological type. There is a celebrated criterion, discovered by J. Douglas [4], [5] (see also [3, Chapter IV] and $[14],[15]$ ), which guarantees the existence of such a minimal surface in certain instances. But this criterion is only a sufficient one; and there are classical examples-the situation of the catenoid being a case in point (see [2, pp. 85-121] and [8]) - which show that minimal surfaces may exist, even though Douglas's condition is violated.

We are concerned here with the special question under which circumstances two distinct Jordan curves $\Gamma_{1}$ and $\Gamma_{2}$ can bound a minimal surface $S$ of the type of the circular annulus. It has recently been shown that the curves $\Gamma_{1}$ and $\Gamma_{2}$ lose this ability if they are too far apart, and quantitative estimates have been given ; $^{2}$ see [1], [8], [9], [10], and [11, pp. 238-241]. If $\Gamma_{1}$ and $\Gamma_{2}$ are sufficiently far apart, they can be separated by a slab bounded by parallel planes. Since each curve is contained in a certain sphere, and since a minimal surface is contained in the convex hull of its boundary (see [12, p. 34]), the intersection of $S$ with the boundary planes of the slab are point sets whose diameter can be estimated in terms of the diameters, or the lengths (if these are finite), of $\Gamma_{1}$ and $\Gamma_{2}$. It is therefore understandable that a discussion of the case where $\Gamma_{1}$ and $\Gamma_{2}$ are curves in parallel planes is of basic importance. It is the purpose of this note to prove the following theorem which, at least by hindsight, is intuitively quite clear:

Let $\Gamma_{1}$ and $\Gamma_{2}$ be two Jordan curves in parallel planes. If there exists a plane which is orthogonal to the planes of these curves and which sepa-

Received by the editors August 10, 1967.

${ }^{1}$ Research sponsored by the Air Force Office of Scientific Research under AFOSR Grant No. 883-67.

${ }_{2}$ The author announced these results at the Joint Soviet-American Symposium on Partial Differential Equations, Novosibirsk, 1963. While the situation for the catenoid, i.e., the case where the coaxial circles $\Gamma_{1}$ and $\Gamma_{2}$ are moved away from each other vertically, has been discussed in the literature many times, it seems strange that the effect of a lateral shift had never been studied before. (Of course, the first case leads to a one-dimensional problem in the calculus of variations.) 
rates them, then $\Gamma_{1}$ and $\Gamma_{2}$ cannot bound a minimal surface of the type of the circular annulus.

Put differently, denoting by $\Gamma_{2}^{1}$ the orthogonal projection of $\Gamma_{2}$ onto the plane of $\Gamma_{1}$ :

If the (closed) convex hulls of $\Gamma_{1}$ and $\Gamma_{2}^{1}$ have no common points, then $\Gamma_{1}$ and $\Gamma_{2}$ cannot bound a minimal surface of the type of the circular annulus.

Note that neither the distance of the planes nor the size of $\Gamma_{1}$ and $\Gamma_{2}$ enter into this statement. Our theorem is sharp in the sense that examples of minimal surfaces exist for which the convex hulls of $\Gamma_{1}$ and $\Gamma_{2}^{1}$ have arbitrarily small intersections of interior points. It also leads to quantitative estimates considerably sharper than the ones obtained before. We finally remark that the simple closed character of the boundary parts $\Gamma_{1}$ and $\Gamma_{2}$ of $S$ is not essential. This can be seen from [10].

For the proof let $\Gamma_{1}$ lie in the plane $z=c_{1}$ and $\Gamma_{2}$ in the plane $z=c_{2}>c_{1}$. Under the assumptions of the theorem, there exist two discs $D_{1}=\left\{x, y, z ;\left(x-x_{1}\right)^{2}+\left(y-y_{1}\right)^{2}<r_{1}^{2}, z=c_{1}\right\}$ and $D_{2}=\left\{x, y, z ;\left(x-x_{2}\right)^{2}\right.$ $\left.+\left(y-y_{2}\right)^{2}<r_{2}^{2}, z=c_{2}\right\}$ containing $\Gamma_{1}$ and $\Gamma_{2}$, respectively, such that $\left(x_{2}-x_{1}\right)^{2}+\left(y_{2}-y_{1}\right)^{2}>\left(r_{1}+r_{2}\right)^{2}$. We shall prove the impossibility of the latter inequality. By the inclusion theorem of [10], if $\Gamma_{1}$ and $\Gamma_{2}$ bound an annulus-type minimal surface $S$, then the boundaries of the discs $D_{1}$ and $D_{2}$, i.e., the circles $\partial D_{1}$ and $\partial D_{2}$, must also bound such a surface $S_{0}$. It has been shown by M. Shiffman [16] that this minimal surface $S_{0}$ is intersected in circles by all planes $z=c\left(c_{1}<c<c_{2}\right)$. As it happens all minimal surfaces generated by a one-parameter family of circles have already been determined in the last century by A. Enneper [6] and B. Riemann [13]. A picture can be found in [7, p. 294]. With the exception of the catenoid these surfaces have a representation in terms of elliptic integrals of the first and second kinds; see [6], [7], [13]. After a suitable choice of the constants, and in a suitable coordinate system, it is

$$
x=\int_{b}^{u} \frac{t^{2} d t}{\Delta(t)}+u \cos v, \quad y=u \sin i, \quad z=a b \int_{b}^{u} \frac{d t}{\Delta(t)} .
$$

Here $0<b \leqq a$ and $\Delta(t)=\left[\left(t^{2}+a^{2}\right)\left(t^{2}-b^{2}\right)\right]^{1 / 2}$. Obviously, $b$ is the radius of the smallest circle, or gorge, on $S_{0}$ which here is contained in the plane $z=0$. (The integrals are two-valued, just as $\cos h^{-1} u$ is.) The curve

$$
x=\int_{b}^{u} \frac{t^{2} d t}{\Delta(t)}, \quad y=0, \quad z=a b \int_{b}^{u} \frac{d t}{\Delta(t)}
$$


denotes the locus of the centers of the generating circles whose radius is equal to $u$. As the $z$-coordinate of this curve varies from $-z_{0}$ to $z_{0}$, where $z_{0}=\left(a b /\left(a^{2}+b^{2}\right)^{1 / 2}\right) \mathrm{K}\left(a /\left(a^{2}+b^{2}\right)^{1 / 2}\right)$, the variable $u$ decreases monotonously from $\infty$ to $b$ and then increases again monotonously from $b$ to $\infty$; see [7].

We now have

$$
\int_{b}^{u} \frac{t^{2} d t}{\Delta(t)}<\int_{b}^{u} \frac{t d t}{\left(t^{2}-b^{2}\right)^{1 / 2}}=\left(u^{2}-b^{2}\right)^{1 / 2}<u .
$$

This inequality has the following interpretation: The lateral distance of the centers of any two of the generating circles is less than the sum of their radii. But this stands in contradiction to the inequality $\left(x_{2}-x_{1}\right)^{2}+\left(y_{2}-y_{1}\right)^{2}>\left(r_{1}+r_{2}\right)^{2}$ from above. The theorem follows from this fact.

\section{Bibilography}

1. P. M. Bailyn, Doubly-connected minimal surfaces, Trans. Amer. Math. Soc. 128 (1967), 206-220.

2. G. A. Bliss, Calculus of variations, Open Court, La Salle, Illinois, 1925.

3. R. Courant, Dirichlet's principle, conformal mapping, and minimal surfaces, Interscience, New York, 1950.

4. J. Douglas, The problem of Plateau for two contours, J. Math. Phys. 10 (1931), 315-359.

5. - The most general form of the problem of Plateau, Amer. J. Math. 61 (1939), 590-608.

6. A. Enneper, Die cyklischen Flächen, Z. Math. Phys. 14 (1869), 393-421.

7. J. C. C. Nitsche, A characterization of the catenoid, J. Math. Mech. 11 (1962), 293-302.

8. - A necessary condition for the existence of certain minimal surfaces, J. Math. Mech. 13 (1964), 659-666.

9. - A supplement to the condition of J. Douglas, Rend. Circ. Mat. Palermo (2) 13 (1964), 192-198.

10. - Ein Einschliessungssatz für Minimalfä̈chen, Math. Ann. 165 (1966), 71-75.

11. - On new results in the theory of minimal surfaces, Bull. Amer. Math. Soc. 71 (1965), 195-270.

12. T. Rad6, On the problem of Plateau, Ergebnisse der Math. 2, Springer, Berlin, 1933.

13. B. Riemann, Ueber die Fläche vom kleinsten Inhalt bei gegebener Begrenzung, Ges. Math. Werke, 2. Aufl., Teubner, Leipzig, 1892, pp. 301-333.

14. M. Shiffman, The Plateau problem for minimal surfaces which are relative minima, Ann. of Math. (2) 39 (1938), 309-315.

UNIVERSITY OF MINNESOTA 